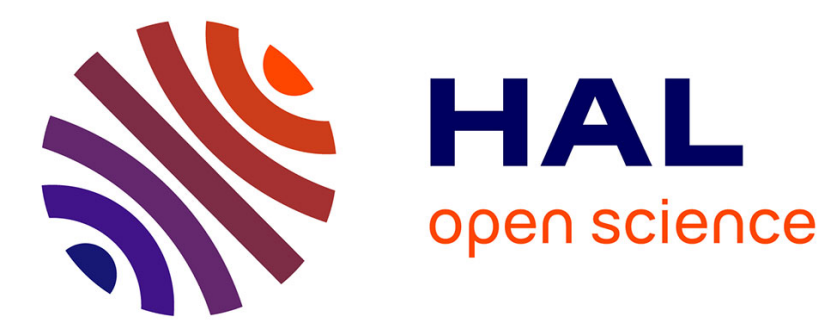

\title{
Robust output stabilization: improving performance via supervisory control
}

Denis Efimov, Antonio Loria, Elena Panteley

\section{To cite this version:}

Denis Efimov, Antonio Loria, Elena Panteley. Robust output stabilization: improving performance via supervisory control. International Journal of Robust and Nonlinear Control, 2011, 21 (10), pp.12191236. 10.1002/rnc.1660 . hal-00521506

\section{HAL Id: hal-00521506 \\ https://hal-centralesupelec.archives-ouvertes.fr/hal-00521506}

Submitted on 28 Mar 2011

HAL is a multi-disciplinary open access archive for the deposit and dissemination of scientific research documents, whether they are published or not. The documents may come from teaching and research institutions in France or abroad, or from public or private research centers.
L'archive ouverte pluridisciplinaire HAL, est destinée au dépôt et à la diffusion de documents scientifiques de niveau recherche, publiés ou non, émanant des établissements d'enseignement et de recherche français ou étrangers, des laboratoires publics ou privés. 


\title{
Robust output stabilization: Improving performance via supervisory control
}

\author{
D. Efimov ${ }^{1}$, A. Loria ${ }^{2, *, \dagger}$ and E. Panteley ${ }^{2}$ \\ ${ }^{1}$ Control of Complex Systems Laboratory, Institute of Problems of Mech. Engg., Bolshoi av., 61, V.O., \\ St-Petersburg 199178, Russia \\ ${ }^{2}$ C.N.R.S, LSS-SUPELEC, Plateau de Moulon, 91192 Gif s/Yvette, France
}

\begin{abstract}
SUMMARY
We analyze robust stability, in an input-output sense, of switched systems. The primary goal (and contribution) of this paper is to design switching strategies to guarantee that input-output stable systems remain so under switching. We propose two types of supervisors: dwell-time and hysteresis based. While our results are stated as tools of analysis they serve a clear purpose in design: to improve performance by appropriately switching among given stabilizing controllers with different performance features. Copyright (C) 2010 John Wiley \& Sons, Ltd.
\end{abstract}

Received 1 October 2009; Revised 19 July 2010; Accepted 23 August 2010

KEY WORDS: supervisory control; input-output stability; performance; ISS; hybrid control; switched systems

\section{INTRODUCTION}

There exist many good reasons and practical motivations to use a set of controllers for a single plant as opposed to one controller: for instance, we may think of a complex system whose dynamic behaviour can be described satisfactorily, from a practical viewpoint, by using several models, each corresponding to a mode of the system or, in other words, each model being valid for state values in a specified region of the state space. In such case, it is common practice to implement a group of 'local controllers applied depending on the operating mode. Another common situation is when design constraints are imposed by relative optimality goals: for instance, one may ask for a controlled plant to track an operating point under constraints regarding transient performance, speed of convergence to the desired operating point, robustness with respect to uncertainties or measurement noise, etc. Therefore, the term optimality shall be understood in that sense: to obtain e.g. the fastest speed of convergence relative to other possibilities but not necessarily to all possibilities.

In such a scenario, it may be convenient to use a set of controllers, each of which achieves the control goal by respecting one of the constraints. Then, given a controller designed to achieve fast convergence and given another one which ensures stabilization with small overshoots, it is desired to design a switching rule between the two controllers to achieve both relatively fast convergence

\footnotetext{
$\overline{{ }^{*} \text { Correspondence }}$ to: A. Loria, C.N.R.S, LSS-SUPELEC, Plateau de Moulon, 91192 Gif s/Yvette, France.

†E-mail: loria@1ss.supelec.fr
} 
at a low price (small overshoots). We address this problem via so-called supervisory control ([1] and $[2$, Chapter 6]).

On a general basis, switching among more than two controllers imposes significant challenges to analysis since classic stability theory does not apply-see [2] and the thorough recent tutorial on hybrid control [3].

While significant advances on stability of hybrid systems have been achieved — see [3, 4] mostly, stability has been regarded in the literature as a property of solutions evolving the state space and most typically with respect to the trivial state motion $\boldsymbol{x}(t) \equiv 0$. However, irrespective of the switching method, the control goal may vary from pure stabilization to robustness with respect to external inputs [5,6] or measurement noise [7] to the more general setting of output stabilization [8]. In such case, the stabilization goal is to bring an output motion close to a desired operating point. Moreover, it appears natural to consider robustness aspects, hence to study stability in an input-output sense. This is the context of our main results.

Roughly speaking, for a given set of 'local' controllers, each achieving a control goal in one dynamic mode, we address the problem of designing a supervisor achieving stability in an input-output sense that is close to that of [9], while improving performance with respect to each controlled mode. Under such setting we show that by appropriate switching (hysteresis-based and dwell-time-based) input-to-output stable systems conserve such property.

The scenario is similar (in spirit) to that studied in the recent paper [10] where the primary goal is to improve performance of switched control systems; in this reference it is assumed that the switching rule is given and the freedom left to the designer resides on setting adequately the initial conditions of the controllers. In the recent tutorial [4] a similar perspective is taken over supervisors, in particular, the notion of covering of the state space is defined along with a switching rule to orchestrate commutations amidst different controllers. However, a fundamental difference with respect to [4] is that in the latter the main concern is 'Lyapunov' stability of equilibria while in this article we make trajectory-based statements to infer input-output properties. In contrast to [4] where the stability proofs are based on dwell-time arguments (the cases of either a common Lyapunov function or of multiple Lyapunov functions with the same values at the instants of switches are considered), the proofs of our main results [11] are trajectory-based and do not impose restrictions on dwell time values.

For instance, the requirement on coincidence of the Lyapunov functions at the instants of switches is relaxed. Another recent article along similar lines is [12] where the authors consider the more particular case of switching between two controlled systems, one global backstepping controller and one local linear-parameter-varying.

The remainder of the paper is organized as follows: Section 2 contains basic definitions and notation; in Section 3 we lay the standing assumptions for our main results, which are presented in Section 4. We present an application of our main results in Section 5 and conclude with some remarks in Section 6.

\section{PRELIMINARIES}

A continuous function $\sigma: \mathbb{R}_{+} \rightarrow \mathbb{R}_{+}$is of class $\mathscr{K}$ if it is strictly increasing and $\sigma(0)=0$; additionally it is of class $\mathscr{K}_{\infty}$ if it is also radially unbounded; a continuous function $\beta: \mathbb{R}_{+} \times \mathbb{R}_{+} \rightarrow \mathbb{R}_{+}$is of class $\mathscr{K} \mathscr{L}$, if $\beta(\cdot, t) \in \mathscr{K}$ for any $t \in \mathbb{R}_{+}$, and $\beta(s, \cdot)$ is strictly decreasing to zero for any $s \in \mathbb{R}_{+}$.

Consider a family of systems

$$
\dot{\boldsymbol{x}}=\boldsymbol{f}_{i}(\boldsymbol{x}, \mathrm{d}), \quad \boldsymbol{y}=\boldsymbol{h}(\boldsymbol{x}), \quad i \in \mathscr{I},
$$

where $\boldsymbol{x} \in \mathbb{R}^{n}$ denotes the state vector; $\mathbf{d} \in \mathbb{R}^{m}$ denotes a disturbance; $\boldsymbol{y} \in \mathbb{R}^{p}$ denotes an output and $i$ is an index taking values from the countable set $\mathscr{I} \subseteq \mathbb{Z}_{+}$. Assume that the functions $\boldsymbol{f}_{i}: \mathbb{R}^{n+l} \rightarrow \mathbb{R}^{n}$, $\boldsymbol{h}: \mathbb{R}^{n} \rightarrow \mathbb{R}^{p}$ are continuous and locally Lipschitz with respect to $\boldsymbol{x}, i \in \mathscr{I}$. It is assumed that $\mathbf{d}: \mathbb{R}_{+} \rightarrow \mathbb{R}^{m}$ is Lebesgue measurable and essentially bounded, for all $t \geqslant 0$

$$
\|\mathbf{d}\|_{[0, t)}=\operatorname{ess} \sup \{|\mathbf{d}(t)|, t \in[0, t)\} .
$$


For $t=+\infty$ we write $\|\mathbf{d}\|=\|\mathbf{d}\|_{[0,+\infty)}$. We denote by $\mathscr{L}_{\infty}^{m}$ the set of functions such that $\|\mathbf{d}\|<+\infty$. Our definition of robust stability is stated in terms of the generalized norm $\mathscr{S}: \mathscr{L}_{\infty}^{m} \times \mathbb{R}^{2} \rightarrow \mathbb{R}_{+}$ defined, for any $t_{0} \geqslant 0$ by

$$
\begin{gathered}
S\left[\mathbf{d}, t_{0}, t\right]:=a \int_{t_{0}}^{t} \omega(|d(\tau)|) \mathrm{d} \tau+b\|\mathbf{d}\|_{\left[t_{0}, t\right)}, \\
a, b \geqslant 0, \quad a+b>0, \quad \omega \in \mathscr{K} .
\end{gathered}
$$

The set of essentially bounded functions $\mathbf{d}$ s.t. $S[d, 0,+\infty]<+\infty$ is denoted as $M_{\mathbb{R}^{m}}$.

Let $i: \mathbb{R}_{+} \rightarrow \mathscr{I}$ be piecewise constant and continuous from the right then, the family of systems (1) defines the following switched system:

$$
\dot{\boldsymbol{x}}=\boldsymbol{f}_{i(t)}(\boldsymbol{x}, \mathbf{d}), \quad \boldsymbol{y}=\boldsymbol{h}(\boldsymbol{x}) .
$$

We say that the switching signal $i(t)$ has an average dwell-time $0<\tau_{\mathrm{D}}<+\infty$ if between switches, for any time instants $t_{2}>t_{1} \geqslant 0$ it holds that

$$
N_{\left[t_{1}, t_{2}\right)} \leqslant N_{0}+\frac{t_{2}-t_{1}}{\tau_{\mathrm{D}}}
$$

for an integer $1 \leqslant N_{0}<+\infty$ and where $N_{\left[t_{1}, t_{2}\right)}$ is the number of discontinuities (switches) of the signal $i(t)$ [13]. If the interval between any two switches is not less than $\tau_{\mathrm{D}}$ then $N_{0}=1$ and the switching signal is said to have dwell-time property. The system (4), for signal $i(t)$ with average dwell-time or simple dwell-time, has a finite number of switches on any finite-time interval and its solution is continuous and defined at least locally [2]. The switched system, for a switching signal $i(t)$, is called forward complete ${ }^{\ddagger}$ if for all initial conditions $\boldsymbol{x}_{0} \in \mathbb{R}^{n}$ and inputs $\mathbf{d} \in M_{\mathbb{R}^{m}}$, the solutions $\boldsymbol{x}\left(t, \boldsymbol{x}_{0}, \mathbf{d}\right)$ of the switched system (4) are defined for all $t \geqslant 0$. We also denote the outputs by $\boldsymbol{y}\left(t, \boldsymbol{x}_{0}, \mathbf{d}\right)=\boldsymbol{h}\left(\boldsymbol{x}\left(t, \boldsymbol{x}_{0}, \mathbf{d}\right)\right)$. On occasions we may use the shorthand notation $\boldsymbol{x}(t)=\boldsymbol{x}\left(t, \boldsymbol{x}_{0}, \mathbf{d}\right)$, $\boldsymbol{y}(t)=\boldsymbol{y}\left(t, \boldsymbol{x}_{0}, \mathbf{d}\right)$.

\section{Definition 1}

We say that for a fixed $i \in \mathscr{I}$ a forward complete system (1) is Input-to-Output Stable (IOS) with respect to the output $\boldsymbol{y}$, the input $\mathbf{d}$ and the norm $\mathscr{S}$ if there exist functions $\beta_{i} \in \mathscr{K} \mathscr{L}, \gamma_{i} \in \mathscr{K}$ such that for all $t \geqslant 0$, all $\boldsymbol{x}_{0} \in \mathbb{R}^{n}$ and $\mathbf{d} \in M_{\mathbb{R}^{m}}$

$$
\left|\boldsymbol{y}\left(t, \boldsymbol{x}_{0}, \mathbf{d}\right)\right| \leqslant \beta_{i}\left(\left|\boldsymbol{x}_{0}\right|, t\right)+\gamma_{i}(\mathscr{S}[\mathbf{d}, 0, t]) .
$$

We say that a switched forward complete system (4) with $i: \mathbb{R}_{+} \rightarrow \mathscr{I}$ is IOS with respect to the output $\boldsymbol{y}$, the input $\mathbf{d}$ and the norm $\mathscr{S}$ if there exist functions $\beta \in \mathscr{K} \mathscr{L}, \gamma \in \mathscr{K}$ such that for all $t \geqslant 0$, all $\boldsymbol{x}_{0} \in \mathbb{R}^{n}$ and $\mathbf{d} \in M_{\mathbb{R}^{m}}$

$$
\left|\boldsymbol{y}\left(t, \boldsymbol{x}_{0}, \mathbf{d}\right)\right| \leqslant \beta\left(\left|\boldsymbol{x}_{0}\right|, t\right)+\gamma(\mathscr{S}[\mathbf{d}, 0, t]) .
$$

\section{Definition 2}

We say that for a fixed $i \in \mathscr{I}$ the forward complete system (1) is state-independent IOS (SIIOS) with respect to the output $\boldsymbol{y}$, the input $\mathbf{d}$ and the norm $\mathscr{S}$ if there exist functions $\beta_{i}^{\prime} \in \mathscr{K} \mathscr{L}, \gamma_{i}^{\prime} \in \mathscr{K}$ such that for all $t \geqslant 0$, all $\boldsymbol{x}_{0} \in \mathbb{R}^{n}$ and $\mathbf{d} \in M_{\mathbb{R}^{m}}$

$$
\left|\boldsymbol{y}\left(t, \boldsymbol{x}_{0}, \mathbf{d}\right)\right| \leqslant \beta_{i}^{\prime}\left(\left|\boldsymbol{h}\left(\boldsymbol{x}_{0}\right)\right|, t\right)+\gamma_{i}^{\prime}(\mathscr{S}[\mathbf{d}, 0, t]) .
$$

We say that the switched forward complete system (4) with $i: \mathbb{R}_{+} \rightarrow \mathscr{I}$ is SIIOS with respect to the output $\boldsymbol{y}$, the input $\mathbf{d}$ and the norm $\mathscr{S}$ if there exist functions $\beta^{\prime} \in \mathscr{K} \mathscr{L}, \gamma^{\prime} \in \mathscr{K}$ such that for all $t \geqslant 0$, all $\boldsymbol{x}_{0} \in \mathbb{R}^{n}$ and $\mathbf{d} \in M_{\mathbb{R}^{m}}$

$$
\left|\boldsymbol{y}\left(t, \boldsymbol{x}_{0}, \mathbf{d}\right)\right| \leqslant \beta^{\prime}\left(\left|\boldsymbol{h}\left(\boldsymbol{x}_{0}\right)\right|, t\right)+\gamma^{\prime}(\mathscr{S}[\mathbf{d}, 0, t]) .
$$

The systems are exponentially SIIOS if $\beta_{i}^{\prime}(s, r)=a s e^{-b r}$ or $\beta^{\prime}(s, r)=a s e^{-b r}$ with $a, b>0$.

\footnotetext{
${ }^{\ddagger}$ Necessary and sufficient conditions for a dynamical system (1) to be forward complete with $S\left[\mathbf{d}, t_{0}, t\right]=\|\mathbf{d}\|_{\left[t_{0}, t\right)}$ can be found in [14].
} 
For the case when $S\left[\mathbf{d}, t_{0}, t\right]=\|\mathbf{d}\|_{\left[t_{0}, t\right)}$, respectively, $S$ is defined by (3), closely connected input-output stability properties and relations between them for nonlinear dynamical systems can be found in [9]; Lyapunov characterizations of these properties are presented in [15].

\section{PROBLEM STATEMENT AND CONTEXT}

Our purpose is to design switching laws to improve performance under the consideration that stabilizing controllers for a given plant are known. By improving performance we understand optimization of convergence rate in the corresponding $\mathscr{K} \mathscr{L}$ estimates. By convergence rate it is meant the time it takes for a curve to reach a given (small) positive value. Certainly, optimization of performance in a conventional setting based on optimization for the solutions themselves is not possible for general nonlinear systems as it is hard to derive the analytical expressions of solutions. Thus, performance improvement is qualitatively measured, not quantitatively.

The following two relaxed hypotheses are needed for the formal statement of our main results. These are statements on stability and not on stabilization hence, the assumptions are expressed in that spirit.

Assumption 1

For each fixed $i \in \mathscr{I}$, the system (1) is forward complete and SIIOS with respect to output $y$ and input $\mathbf{d}$ with functions $\beta_{i} \in \mathscr{K} \mathscr{L}, \gamma_{i}:=\gamma \in \mathscr{K}$ and norm $\mathscr{S}$.

From Definition 2 it holds that $\beta_{i}(s, 0) \geqslant s$ for all $s \geqslant 0$. Without loss of generality we assume that $\beta_{i}(s, 0)>s, s>0$ and denote $\chi_{i}(s)=\beta_{i}^{-1}(s, 0)$.

Technically speaking, Assumption 1 states that we are given $N$ (closed-loop) systems that are SIIOS. In other words, in the context of control design, it states that we dispose of $N$ stabilizing controllers.

The second standing hypothesis states that the control designer has made a partition of the real line, relative to the values of interest of the norm of the output to be controlled. Such partition may come e.g. from a look-up table constructed on the basis of different models and controllers for distinct linearizations of the plant over different operating points. The look-up table may also be constructed based on the performance of a determined controller: for instance, high control gain may be desirable for large errors while highly damped controllers may be more attractive in a neighbourhood of the equilibrium.

More precisely, let $N$ be an integer that denotes the number of controllers, let $M$ be a positive integer that denotes the number of stability regions of interest (partitions of the real line); let $q \in\{0, \ldots, M\}$ define the strictly increasing sequence $\left\{\Delta_{q}\right\}$ and let each system from the family (1) be labeled $\theta_{q} \in \mathscr{I}$ for each such $q$; let $T_{q}$ denote a dwell-time function, i.e. each system is active for at least $T_{q}$ units of time.

Assumption 2

A partition $\mathbb{R}_{+}=\bigcup_{q=0}^{M}\left[\Delta_{q}, \Delta_{q+1}\right), \Delta_{0}=0, \Delta_{M+1}=+\infty$ is given and for each $q \in\{0, \ldots, M\}$ there exists $\theta_{q} \in \mathscr{I}$ such that $\beta_{\theta_{q}}\left(\Delta_{q}, 0\right) \leqslant \Delta_{q+1}, \quad \beta_{\theta_{q}}\left(\Delta_{q+1}, 0\right) \leqslant \Delta_{q+2}$. Furthermore, let $T_{q}$ : $\left[\Delta_{q}, \Delta_{q+1}\right) \rightarrow \mathbb{R}_{+}$be given continuous, bounded and separated-from-zero, i.e. $0<T_{\min }=$ $\min _{0 \leqslant q \leqslant M}\left\{\inf _{\Delta_{q} \leqslant s<\Delta_{q+1}}\left\{T_{q}(s)\right\}\right\}<+\infty$.

A graphical illustration of Assumption 2 is presented in Figure 1. We depict a possible partition of $\mathbb{R}_{+}$relative to values of $|\boldsymbol{y}|$ that are considered of interest in the control design. In general, this depends on the case-study and therefore, the partition is not fixed by assumption. The figure illustrates a case in which controller 1 is preferred to others for small output values hence $\theta_{0}=1$, while controller 2 is used for large error values contained in the interval $\left[\Delta_{M-1}, \Delta_{M}\right)$ hence $\theta_{M-1}=2$. For illustration, suppose that it is desired to obtain 'fast convergence with 'small' overshoots. Assumption 2 holds, for instance, if $\beta_{\theta_{q}}\left(s, T_{q}(s)\right) \leqslant \beta_{i}\left(s, T_{q}(s)\right)$ for all $s \in\left[\Delta_{q}, \Delta_{q+1}\right)$, $i \in \mathscr{I}$, which implies that the estimate on output trajectories of the system $\theta_{q}$, starting off in the 


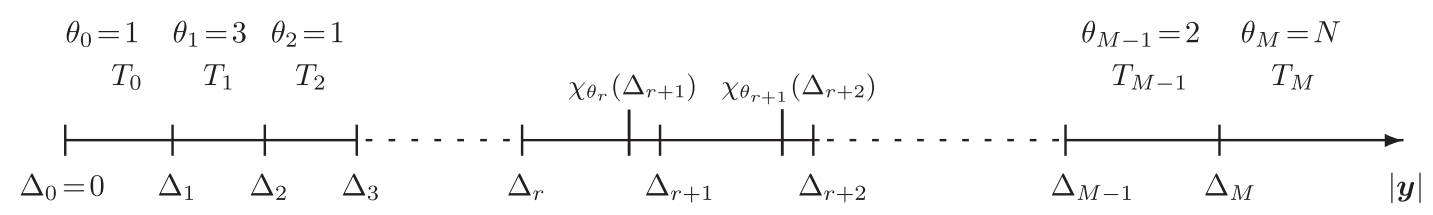

Figure 1. Illustrative example when Assumption 2 holds.

interval $\left[\Delta_{q}, \Delta_{q+1}\right)$, converge the fastest to zero relative to the output estimates of any other closedloop system in (1). It is left to design an appropriate switching among the controllers to guarantee fastest convergence for the output of (1) subject to a small-overshoots constraint.

The restriction $\beta_{\theta_{q}}\left(\Delta_{q+1}, 0\right) \leqslant \Delta_{q+2}$ implies that without disturbances the system output trajectory contained in the interval $\left[\Delta_{q}, \Delta_{q+1}\right)$ can only enter the neighbouring intervals. The property $\beta_{\theta_{q}}\left(\Delta_{q}, 0\right) \leqslant \Delta_{q+1}$ implies that any interval $\left[\Delta_{q}, \Delta_{q+1}\right)$ can be decomposed on two nonempty subsets $\left[\Delta_{q}, \chi_{\theta_{q}}\left(\Delta_{q+1}\right)\right)$ and $\left[\chi_{\theta_{q}}\left(\Delta_{q+1}\right), \Delta_{q+1}\right)$, the latter one plays a role of hysteresis. These properties are used for purposes of proof.

\section{SUPERVISORY OUTPUT CONTROL}

\subsection{Dwell-time supervisor}

Informally speaking, the dwell-time supervisor is defined as follows: consider a set of IOS systems labeled $\theta_{q}$ with $q \in\{0, \ldots, M\}$ and a given partition of the non-negative real line as described in Assumption 2 and illustrated in Figure 1. After a given instant $t_{j}$ at which the output trajectory lays in the interval $\left[\Delta_{j}, \Delta_{j+1}\right)$ the next switching instant is determined as the earliest moment, passed a dwell-time, such that the output in norm strictly belongs to a partition $\left[\Delta_{k}, \Delta_{k+1}\right)$ with $k \neq j$. In view of the dwell-time the $k$ th and the $j$ th intervals are in no particular order with respect to each other and are not necessarily contiguous; e.g. the output trajectory $|\boldsymbol{y}(t)|$ may pass from the interval $\left[\Delta_{1}, \Delta_{2}\right)$ to the interval $\left[\Delta_{5}, \Delta_{6}\right)$ or vice-versa by 'crossing' intermediate intervals during the dwell time. Once the switching instant is identified, the switching rule (the supervisor) takes the value corresponding to the interval in which the switch occurs; i.e. the $k$ th mode is selected.

More precisely, the dwell-time supervisor is defined as follows:

$$
\begin{gathered}
t_{0}=0, \quad i\left(t_{0}\right)=r, \quad r \in\{0, \ldots, M\},\left|\boldsymbol{h}\left(\boldsymbol{x}\left(t_{0}\right)\right)\right| \in\left[\Delta_{r}, \Delta_{r+1}\right) \\
t_{j+1}=\min \left\{t \geqslant t_{j}+T_{i\left(t_{j}\right)}, t:|h(x(t))| \in \bigcup_{q=0, q \neq i\left(t_{j}\right)}^{M}\left[\Delta_{q}, \chi_{\theta_{q}}\left(\Delta_{q+1}\right)\right)\right\} \\
i\left(t_{j+1}\right)=k \quad \text { such that }\left|\boldsymbol{h}\left(\boldsymbol{x}\left(t_{j+1}\right)\right)\right| \in\left[\Delta_{k}, \chi_{\theta_{k}}\left(\Delta_{k+1}\right)\right) \subset\left[\Delta_{k}, \Delta_{k+1}\right) .
\end{gathered}
$$

The dwell-time depends on the output trajectories, i.e. $T_{i\left(t_{j}\right)}=T_{i\left(t_{j}\right)}\left(\left|\boldsymbol{h}\left(\boldsymbol{x}\left(t_{j}\right)\right)\right|\right), t_{j}$ are switching times; $j=1,2,3, \ldots$ is the index of the last switch and the signal $i(t)$ is piecewise constant for all $t$ such that $\left|\boldsymbol{h}\left(\boldsymbol{x}\left(t_{j+1}\right)\right)\right| \in \mathscr{N}=\bigcup_{q=0}^{M}\left[\chi_{\theta_{q}}\left(\Delta_{q+1}\right), \Delta_{q+1}\right)$. The introduction of $\chi_{\theta_{i\left(t_{j}\right)}}$ prevents from fast switching (chattering) and plays the role of hysteresis [13,16]. The second mechanism that prevents the system from chattering is output ${ }^{\S}$ dependent dwell-time $T_{i\left(t_{j}\right)}$. Since by construction the functions $T_{q}, q \in\{0, \ldots, M\}$ are bounded and separated from zero the system (4), with supervisor (5a), has dwell-time $T_{q}(s) \geqslant T_{\min }$ and the system undergoes a finite number of switches on any finite-time interval.

\footnotetext{
${ }^{\S}$ In this respect, we mention in [17] where dwell-time depends on the whole state as opposed to an output of the system.
} 


\section{Remark 1}

In contrast to the state regulation problem-see $[18,19]$, in the present context it does not suffice in general to introduce hysteresis to guarantee a finite number of switches. This is because the output regulation set $\mathscr{N}$ may be noncompact. This stymies the estimation of the system's behaviour on the set e.g. it is possible that a system's state trajectory (in norm) escapes to infinity while the output remains in $\mathscr{N}$. In Section 4.2 a hysteresis supervisor is studied in detail further.

\section{Remark 2}

Note that Assumption 2 can be relaxed for the supervisor (5a). The function $T_{0}$ can be chosen identically equal to zero. In that case the system has average dwell-time $T_{\min }$ with $N_{0}=2$. According to $(5 \mathrm{a}), i\left(t_{j}\right) \neq i\left(t_{j+1}\right)$ so the system $\theta_{0}$ may become active each second time only. Therefore, the time interval between two switches is bigger than $T_{\min }$. In this case, the time $T_{\min }$ is calculated for $q \in\{0, \ldots, M\}$.

\section{Theorem 1}

Let Assumptions 1 and 2 hold. Then, the system (4) with supervisor (5a), measurable disturbances $\mathbf{d} \in M_{\mathbb{R}^{m}}$ and initial conditions $t_{0}=0, \boldsymbol{x}_{0} \in \mathbb{R}^{n}$,

(i) is forward complete and, for all $t \geqslant 0$,

$$
\begin{aligned}
\left|\boldsymbol{y}\left(t, \boldsymbol{x}_{0}, \mathbf{d}\right)\right| & \leqslant \bar{\beta}\left(\max \left\{2 \Delta_{M},\left|\boldsymbol{h}\left(\boldsymbol{x}_{0}\right)\right|\right\}, 0\right)+\bar{\gamma}(\mathscr{S}[\mathbf{d}, 0, t]) ; \\
\bar{\beta}(s, 0) & :=\sup _{i \in \mathscr{I}} \beta_{i}(s, 0) ; \\
\bar{\gamma}(s) & :=\gamma(s)+\beta_{\theta_{M}}(2 \gamma(s), 0)
\end{aligned}
$$

(ii) furthermore, if the system dynamics undergoes a finite number of switches over infinite time, i.e. if there exists $N<\infty$ such that $\left\{t_{k}\right\} \rightarrow t_{N}<\infty$ (define $t_{N+1}:=+\infty$ ) then, there exists a continuous function $\bar{\beta}^{\mathbf{d}}: \mathbb{R}_{+} \times \mathbb{R}_{+} \rightarrow \mathbb{R}_{+}$such that $\bar{\beta}^{\mathbf{d}}(\cdot, s)$ is strictly increasing for each $s$ and $\bar{\beta}^{\mathbf{d}}(r, \cdot)$ is strictly decreasing to zero for each $r$, such that ${ }^{\mathbb{t}}$

$$
\left|\boldsymbol{y}\left(t, \boldsymbol{x}_{0}, \mathbf{d}\right)\right| \leqslant \bar{\beta}^{\mathbf{d}}\left(\left|\boldsymbol{h}\left(\boldsymbol{x}_{0}\right)\right|, t\right)+\gamma(S[\mathbf{d}, 0, \infty]) ;
$$

(iii) finally, if $\mathbf{d}(t) \equiv 0$ (with no assumption on the number of switchings) the output trajectories $\boldsymbol{y}(t)$ satisfy

$$
\left|\boldsymbol{y}\left(t, \boldsymbol{x}_{0}, \mathbf{d}\right)\right| \leqslant \bar{\beta}^{\mathbf{0}}\left(\left|\boldsymbol{h}\left(\boldsymbol{x}_{0}\right)\right|, t\right),
$$

where $\bar{\beta}^{\mathbf{0}} \in \mathscr{K} \mathscr{L}$.

Proof of $(i)$.

On any time interval $\left[T_{s}, T_{e}\right)$ with $T_{e}>T_{s} \geqslant 0$ a finite number of switches $N_{\left[T_{s}, T_{e}\right)}$ with the upper estimate

$$
N_{\left[T_{s}, T_{e}\right)} \leqslant \frac{1+\left(T_{e}-T_{S}\right)}{T_{\min }}
$$

may occur. Between switches, the system's dynamics is continuous and is equivalent to that of a system from the family (1) and which is forward complete for $i \in I$ fixed arbitrarily. Since the signal $i(t)$ remains piecewise constant over $\left[T_{s}, T_{e}\right.$ ) the solutions of system (4), (5a) are continuous and are defined at least locally on the same interval. From continuity of solutions of (4), (5a) and forward completeness of $\dot{\boldsymbol{x}}=\boldsymbol{f}_{i}(\boldsymbol{x}, \mathbf{d})$ with fixed $i$, it follows that solutions of (4), (5a) are also defined at $T_{e}$. The same arguments hold for any interval $\left[T_{s}, T_{e}\right)$ therefore the switched system (4), (5a) is forward complete.

\footnotetext{
IThat is, $\bar{\beta}^{\text {d }}$ is 'of class $\mathscr{K} \mathscr{L}$ ' with the exception that $\bar{\beta}^{\mathbf{d}}(0, \cdot)$ may be different from zero.
} 
We prove next that the bound (6a) holds. From (5a) we have $i(0)=r$, where $r \in\{0, \ldots, M\}$ and $\left|\boldsymbol{h}\left(\boldsymbol{x}_{0}\right)\right| \in\left[\Delta_{r}, \Delta_{r+1}\right)$. According to Assumption 1, in this case,

$$
\left|\boldsymbol{y}\left(t, \boldsymbol{x}_{0}, \mathbf{d}\right)\right| \leqslant \beta_{\theta_{r}}\left(\left|\boldsymbol{h}\left(\boldsymbol{x}_{0}\right)\right|, t\right)+\gamma\left(S\left[\mathbf{d}, 0, t_{1}\right]\right) \quad \forall t \in\left[0, t_{1}\right)
$$

where $t_{1}$ is the first switching time and, according to $(5 \mathrm{a}), t_{1}$ is when the output trajectory belongs to the interval $\left[\Delta_{i\left(t_{1}\right)}, \chi_{\theta_{i\left(t_{1}\right)}}\left(\Delta_{i\left(t_{1}\right)+1}\right)\right)$ with $i\left(t_{1}\right) \in\{0, \ldots, M\}$ and $i\left(t_{1}\right) \neq i(0)$. If $t_{1}=+\infty$ the proof ends replacing the bound in (6a) with

$$
\left|\boldsymbol{y}\left(t, \boldsymbol{x}_{0}, \mathbf{d}\right)\right| \leqslant \beta_{\theta_{r}}\left(\left|\boldsymbol{h}\left(\boldsymbol{x}_{0}\right)\right|, 0\right)+\gamma(S[\mathbf{d}, 0, \infty]) \quad \forall t \geqslant 0 .
$$

Otherwise, if $t_{1}<+\infty$, by continuity of solutions, continuity of $\boldsymbol{h}$ and forward completeness the solutions may be continued up to $t_{1}$ and

$$
\left|\boldsymbol{y}\left(t_{1}\right)\right| \leqslant \chi_{\theta_{i\left(t_{1}\right)}}\left(\Delta_{i\left(t_{1}\right)+1}\right) .
$$

Reconsidering the solutions with new 'initial' condition $t_{1}, x\left(t_{1}\right)$ we obtain, from the definition of the supervisor $(5 \mathrm{a})$

$$
\left|\boldsymbol{y}\left(t, \boldsymbol{x}\left(t_{1}\right), \mathbf{d}\right)\right| \leqslant \beta_{\theta_{i\left(t_{1}\right)}}\left(\left|\boldsymbol{y}\left(t_{1}\right)\right|, t-t_{1}\right)+\gamma\left(S\left[\mathbf{d}, t_{1}, t_{2}\right]\right), \quad t \in\left[t_{1}, t_{2}\right) .
$$

Using (10) and the definition of $\chi_{\theta_{i\left(t_{1}\right)}}$ in the expression above, we obtain

$$
\left|\boldsymbol{y}\left(t, \boldsymbol{x}\left(t_{1}\right), \mathbf{d}\right)\right| \leqslant \Delta_{i\left(t_{1}\right)+1}+\gamma\left(S\left[\mathbf{d}, t_{1}, t_{2}\right]\right), \quad t \in\left[t_{1}, t_{2}\right) .
$$

By definition, at time instant $t_{2}$ the system's output reaches for the interval $\left[\Delta_{i\left(t_{2}\right)}, \chi_{\theta_{i\left(t_{2}\right)}}\left(\Delta_{i\left(t_{2}\right)+1}\right)\right)$ with $i\left(t_{2}\right) \in\{0, \ldots, M\}$ and $i\left(t_{2}\right) \neq i\left(t_{1}\right)$ and $\left|\boldsymbol{y}\left(t_{2}\right)\right| \leqslant \chi_{\theta_{i\left(t_{2}\right)}}\left(\Delta_{i\left(t_{2}\right)+1}\right)$. Following similar arguments as above we see that

$$
\left|\boldsymbol{y}\left(t, \boldsymbol{x}\left(t_{2}\right), \mathbf{d}\right)\right| \leqslant \Delta_{i\left(t_{2}\right)+1}+\gamma\left(S\left[\mathbf{d}, t_{2}, t_{3}\right]\right), \quad t \in\left[t_{2}, t_{3}\right) .
$$

Repeating the previous steps we obtain, for arbitrary time instants $t_{j}$ for any $j>1$

$$
\begin{gathered}
\left|\boldsymbol{y}\left(t, \boldsymbol{x}\left(t_{j}\right), \mathbf{d}\right)\right| \leqslant \Delta_{i\left(t_{j}\right)+1}+\gamma\left(S\left[\mathbf{d}, t_{j}, t_{j+1}\right]\right), \quad t \in\left[t_{j}, t_{j+1}\right), \\
\left|\boldsymbol{y}\left(t_{j}\right)\right| \leqslant \chi_{\theta_{i\left(t_{j}\right)}}\left(\Delta_{i\left(t_{j}\right)+1}\right) .
\end{gathered}
$$

Now, if at some step $k$ the system's output (in norm) belongs to the last interval $\left[\Delta_{M}, \Delta_{M+1}\right.$ ) with $\Delta_{M+1}=+\infty$ then, the maximum output amplitude may be estimated by

$$
|\boldsymbol{y}(t)| \leqslant \beta_{\theta_{M}}\left(\left|\boldsymbol{y}\left(t_{k}\right)\right|, 0\right)+\gamma\left(S\left[\mathbf{d}, t_{k}, t_{k+1}\right]\right), \quad t \in\left[t_{k}, t_{k+1}\right) .
$$

According to (11) the possible values of $\boldsymbol{y}\left(t_{k}\right)$ are bounded byll

$$
\left|\boldsymbol{y}\left(t_{k}\right)\right| \leqslant \Delta_{M}+\gamma(S[\mathbf{d}, 0, t])
$$

Therefore, for all $t \in\left[t_{k}, t_{k+1}\right)$,

$$
|\boldsymbol{y}(t)| \leqslant \beta_{\theta_{M}}\left(\Delta_{M}+\gamma(S[\mathbf{d}, 0, t]), 0\right)+\gamma(S[\mathbf{d}, 0, t]) .
$$

By induction, the latter holds for any integer $k \geqslant 1$ hence, for all $t \geqslant t_{1}$. Using the triangle inequality $\beta_{\theta_{M}}(a+b, 0) \leqslant \beta_{\theta_{M}}(2 a, 0)+\beta_{\theta_{M}}(2 b, 0)$ and the bound (9) we obtain (6a).

Proof of (ii).

We derive next a bound for the output trajectories which leads to (7). Following the definition of the supervisor (5a) we have (9). According to (5a), $t_{1}$ is the first switching time, i.e. when the

"Where in view of the definition of the supervisor (5b) we exclude the value $i\left(t_{k}\right)+1=M+1=\infty$. 
norm of the output trajectory belongs to the interval $\left[\Delta_{i\left(t_{1}\right)}, \chi_{\theta_{i\left(t_{1}\right)}}\left(\Delta_{i\left(t_{1}\right)+1}\right)\right)$ with $i\left(t_{1}\right) \in\{0, \ldots, M\}$ and $i\left(t_{1}\right) \neq i(0)$. If $t_{1}=+\infty$ the proof ends since (9) implies (7).

Otherwise, if $t_{1}<+\infty$ by continuity of solutions, continuity of $\boldsymbol{h}$, forward completeness, and the definition of the dwell-time function $T_{i}\left(t_{1}\right)$ in Assumption 2, we have

$$
\left|\boldsymbol{y}\left(t_{1}\right)\right| \leqslant \beta_{\theta_{r}}\left(\left|\boldsymbol{h}\left(\boldsymbol{x}_{0}\right)\right|, T_{\min }\right)+\gamma\left(S\left[\mathbf{d}, 0, t_{1}\right]\right) .
$$

Under similar arguments let $t_{2}$ be the second switching instant, that is the first time such that $|\boldsymbol{y}(t)| \in\left[\Delta_{i\left(t_{2}\right)}, \chi_{\theta_{i\left(t_{2}\right)}}\left(\Delta_{i\left(t_{2}\right)+1}\right)\right)$ with $0 \leqslant i\left(t_{2}\right) \leqslant M$ and $i\left(t_{2}\right) \neq i\left(t_{1}\right)$. We have

$$
|\boldsymbol{y}(t)| \leqslant \beta_{\theta_{i\left(t_{1}\right)}}\left(\left|\boldsymbol{y}\left(t_{1}\right)\right|, t-t_{1}\right)+\gamma\left(S\left[\mathbf{d}, t_{1}, t_{2}\right]\right) \quad \forall t \in\left[t_{1}, t_{2}\right)
$$

and, if $t_{2}<+\infty$,

$$
\left|\boldsymbol{y}\left(t_{2}\right)\right| \leqslant \beta_{\theta_{i\left(t_{1}\right)}}\left(\left|\boldsymbol{y}\left(t_{1}\right)\right|, T_{\min }\right)+\gamma\left(S\left[\mathbf{d}, t_{1}, t_{2}\right]\right) .
$$

Using (15) in (14) we obtain

$$
\begin{aligned}
& |\boldsymbol{y}(t)| \leqslant \beta_{\theta_{i\left(t_{1}\right)}}\left(\beta_{\theta_{r}}\left(\left|\boldsymbol{h}\left(\boldsymbol{x}_{0}\right)\right|, T_{\min }\right)+\gamma\left(S\left[\mathbf{d}, 0, t_{1}\right]\right), t-t_{1}\right)+\gamma\left(S\left[\mathbf{d}, t_{1}, t_{2}\right]\right) \quad \forall t \in\left[t_{1}, t_{2}\right), \\
& \left|\boldsymbol{y}\left(t_{2}\right)\right| \leqslant \beta_{\theta_{i\left(t_{1}\right)}}\left(\beta_{\theta_{r}}\left(\left|\boldsymbol{h}\left(\boldsymbol{x}_{0}\right)\right|, T_{\min }\right)+\gamma\left(S\left[\mathbf{d}, 0, t_{1}\right]\right), T_{\min }\right)+\gamma\left(S\left[\mathbf{d}, t_{1}, t_{2}\right]\right) .
\end{aligned}
$$

Similar arguments hold for any $k \geqslant 1$ and all $t \in\left[t_{k-1}, t_{k}\right)$, i.e.

$$
\begin{aligned}
|\boldsymbol{y}(t)| \leqslant & \beta_{\theta_{i\left(t_{k-1}\right)}}\left(\beta_{\theta_{i\left(t_{k-2}\right)}} \circ\left(\cdots \circ\left(\vartheta_{0}^{\mathbf{d}}\left(\left|\boldsymbol{h}\left(x_{0}\right)\right|\right), T_{\min }\right)+\cdots+\gamma\left(S\left[\mathbf{d}, t_{k-2}, t_{k-1}\right]\right)\right), t-t_{k-1}\right) \\
& +\gamma\left(S\left[\mathbf{d}, t_{k-1}, t_{k}\right]\right), \\
\vartheta_{0}^{\mathbf{d}}(s):= & \beta_{\theta_{r}}\left(s, T_{\min }\right)+\gamma\left(S\left[\mathbf{d}, 0, t_{1}\right]\right) .
\end{aligned}
$$

By assumption there exists $N<\infty$ such that $\left\{t_{k}\right\} \rightarrow t_{N}<\infty$ and $t_{N+1}:=+\infty$; from this and (19) it follows that, for all $t \in\left[t_{k}, \infty\right)$,

$$
\begin{aligned}
|\boldsymbol{y}(t)| \leqslant \widetilde{\beta}^{\mathbf{d}}\left(\left|\boldsymbol{h}\left(\boldsymbol{x}_{0}\right)\right|, t-t_{k}\right)+\gamma\left(S\left[\mathbf{d}, t_{k}, \infty\right]\right) \\
\left.\tilde{\beta}^{\mathbf{d}}(s, r):=\beta_{\theta_{i\left(t_{k}\right)}}(\beta_{\theta_{i\left(t_{k-1}\right)}} \overbrace{\circ(\cdots \circ}^{N \text { times }}\left(\vartheta_{0}^{\mathbf{d}}(s), T_{\min }\right)+\cdots+\gamma(S[\mathbf{d}, 0, \infty])), r\right) .
\end{aligned}
$$

The function $\widetilde{\beta}^{\mathbf{d}}(\cdot, r)$ is strictly increasing for each fixed $r$ and $\bar{\beta}^{\mathbf{d}}(s, \cdot)$ is strictly decreasing to zero for each fixed $s$.

Define $\kappa^{\mathbf{d}}(s)$ as the right-hand side of $(6 a)$, i.e. $\kappa^{\mathbf{d}}(s):=\bar{\beta}\left(\max \left\{2 \Delta_{M}, s\right\}, 0\right)+\bar{\gamma}(\mathscr{S}[\mathbf{d}, 0, \infty])$. Then, $\bar{\beta}^{\mathbf{d}}$ may be defined as any continuous function satisfying the following: $\bar{\beta}(\cdot, r)$ is strictly increasing for each $r \geqslant 0 ; \bar{\beta}^{\mathbf{d}}(s, \cdot)$ is strictly decreasing for each $s \geqslant 0$

$$
\begin{aligned}
\bar{\beta}^{\mathbf{d}}\left(s, t_{N}\right) & =\kappa^{\mathbf{d}}(s) ; \\
\bar{\beta}^{\mathbf{d}}(s, t) & \geqslant \widetilde{\beta}^{\mathbf{d}}\left(s, t-t_{N}\right) \quad \forall t \geqslant t_{N} ; \\
\bar{\beta}^{\mathbf{d}}(s, t) & :=\beta^{*}(s, t) \in \mathscr{K} \mathscr{L} \quad \forall t \in\left[0, t_{N}\right] ;
\end{aligned}
$$

such that $\beta^{*}\left(s, t_{N}\right)=\kappa^{\mathbf{d}}(s)$.

Proof of (iii).

Consider $\mathbf{d} \equiv 0$ and let the initial conditions be $i(0)=r$ with $r \in\{0, \ldots, M\}$, where $|\boldsymbol{h}(\mathbf{x}(0))| \in$ $\left[\Delta_{r}, \Delta_{r+1}\right)$. If $|\boldsymbol{h}(\mathbf{x}(0))| \in\left[\chi_{\theta_{r}}\left(\Delta_{r+1}\right), \Delta_{r+1}\right)$, the output trajectory $|\boldsymbol{y}(t)|$ may, in general, reach the interval $\left[\Delta_{r+1}, \chi_{\theta_{r+1}}\left(\Delta_{r+2}\right)\right)$. On the other hand, if $|\boldsymbol{h}(\mathbf{x}(0))| \in\left[\Delta_{r}, \chi_{\theta_{r}}\left(\Delta_{r+1}\right)\right)$ for any $r$ we have, by assumption, $|\boldsymbol{y}(t)| \leqslant \beta_{\theta_{r}}(|\boldsymbol{y}(0)|, 0)$ which implies that $|\boldsymbol{y}(t)|<\Delta_{r+1}$, i.e. the output trajectory (in 
norm) cannot reach higher intervals than $\left[\Delta_{r+1}, \chi_{\theta_{r+1}}\left(\Delta_{r+2}\right)\right)$ and may only decrease for at least $T_{\theta_{r}}$ units of time. Therefore, there exists $t^{\prime}$ such that the system's output trajectories reach the lower next interval $\left[\Delta_{r-1}, \chi_{\theta_{r-1}}\left(\Delta_{r}\right)\right)$. Repeating the reasoning we conclude that $|\boldsymbol{y}(t)|$ may only continue decreasing to zero (right to left on the real line -see Figure 1) which implies that the system (1) can undergo only a finite number of switches over $[0, \infty)$. The proof ends.

The theorem establishes boundedness of the output trajectories for any bounded disturbances. Without disturbances, the output converges to zero. Note that the stability estimate depends on the partition, this prevents us from computing a $\mathscr{K} \mathscr{L}$ estimate on the overall supervisor solutions. This is a structural feature related to the supervisor construction; under the effect of disturbances, a trajectory may reach any interval of the partition.

\section{Remark 3}

The partitioning of the real line as showed in Figure 1 is reminiscent of the so-called covering method from [4] where stability is studied in the state space as opposed to the output space. In contrast to the latter reference dwell-time functions $T_{q}:\left[\Delta_{q}, \Delta_{q+1}\right) \rightarrow \mathbb{R}_{+}, q \in\{0, \ldots, M\}$ are used merely to impose a delay between switches thereby, to imply right-continuity of the switching signal $i(t)$ and, hence, the system solutions. Since the functions $T_{q}$ define an output-dependent dwell-time the latter differs for each subsystem in (1). However, we do not use dwell-time arguments to imply stability hence, no other restriction than $T_{q}$ taking values separated from zero is imposed in the assumption.

\subsection{Hysteresis supervisor}

We now trade the dwell-time condition by a hysteresis assumption. On the contrary to the previous case in which switches may occur to any mode provided that a minimal time passes, we now assume that switches occur as soon as the output value (in norm) leaves a determined interval modulo a hysteresis zone to prevent infinite switches over finite intervals. In particular, switching may occur from mode $q$ to modes $q-1$ or $q+1$ only. The hysteresis supervisor is defined as follows:

$$
\begin{aligned}
t_{0} & =0, \quad i\left(t_{0}\right)=r, \quad r \in\{0, \ldots, M\},\left|\boldsymbol{h}\left(\boldsymbol{x}\left(t_{0}\right)\right)\right| \in\left[\Delta_{r}, \Delta_{r+1}\right) ; \\
t_{j+1} & =\min \left\{t \geqslant t_{j}:|\boldsymbol{h}(\boldsymbol{x}(t))| \in\left[\Delta_{k}, \chi_{\theta_{k}}\left(\Delta_{k+1}\right)\right), \quad k \in\left\{i\left(t_{j}\right)-1, i\left(t_{j}\right)+1\right\}\right\} \\
i\left(t_{j+1}\right) & =k \quad \text { such that }\left|\boldsymbol{h}\left(\boldsymbol{x}\left(t_{j+1}\right)\right)\right| \in\left[\Delta_{k}, \chi_{\theta_{k}}\left(\Delta_{k+1}\right)\right) \subset\left[\Delta_{k}, \Delta_{k+1}\right) .
\end{aligned}
$$

where $t_{j}$, with $j=1,2,3, \ldots$, are switching times; $j$ is the number of the last switch and the signal $i(t)$ has constant value in the so-called 'hysteresis' set $\mathscr{N}=\bigcup_{q=0}^{M-1}\left[\chi_{\theta_{q}}\left(\Delta_{q+1}\right), \Delta_{q+1}\right)$.

\section{Theorem 2}

Let Assumptions 1 and 2 hold. Then, for the system (4) with supervisor (23a), measurable disturbances $\mathbf{d} \in M_{\mathbb{R}^{m}}$ and initial conditions $t_{0}=0, \boldsymbol{x}_{0} \in \mathbb{R}^{n}$, items (i)-(iii) of Theorem 1 hold with

$$
\left|\boldsymbol{y}\left(t, \boldsymbol{x}_{0}, \mathbf{d}\right)\right| \leqslant \bar{\beta}\left(\max \left\{\Delta_{M},\left|\boldsymbol{h}\left(\boldsymbol{x}_{0}\right)\right|\right\}, 0\right)+\gamma(\mathscr{S}[\mathbf{d}, 0, t]) \quad \forall t \geqslant t_{0},
$$

instead of (6a).

\section{Remark 4}

It is important to stress that in the previous statement Assumption 1 needs not to hold globally, i.e. each of the systems in (4) is not required to be SIIOS for all initial states and all measurable disturbances. It is sufficient that each system $\theta_{q}$ is SIIOS for initial states in the set corresponding to output values where the system is to be active. To illustrate this, consider Figure 1 and system $\theta_{1}$; for the hysteresis supervisor it is enough that the system $\theta_{1}$ be SIIOS for all $\boldsymbol{x}_{0}$ such that $\left|\boldsymbol{h}\left(\boldsymbol{x}_{0}\right)\right| \in\left[\Delta_{0}, \Delta_{1}\right] \cup\left[\Delta_{2}, \Delta_{3}\right]$. This is of obvious interest if system $\theta_{1}$ corresponds to a plant in closed loop with a controller which guarantees stability in the large (i.e. in a 'large' specified domain of attraction, subset of $\mathbb{R}^{n}$ ) or even locally. See [11] for some examples. 
Proof of Theorem 2

From (23a) $i\left(t_{0}\right)=r, t_{0}=0$, where $\left|\boldsymbol{h}\left(\boldsymbol{x}_{0}\right)\right| \in\left[\Delta_{r}, \Delta_{r+1}\right), r \in\{0, \ldots, M\}$. According to Assumption 1 system $\theta_{r}$ from family (1) is forward complete so estimate

$$
\left|\boldsymbol{y}\left(t, \boldsymbol{x}_{0}, \mathbf{d}\right)\right| \leqslant \beta_{\theta_{i\left(t_{0}\right)}}\left(\left|\boldsymbol{h}\left(\boldsymbol{x}_{0}\right)\right|, t\right)+\gamma\left(S\left[\mathbf{d}, 0, t_{1}\right]\right)
$$

holds also for the trajectories of system (4), (23a) over $t \in\left[t_{0}, t_{1}\right)$. From (23a) the time instant $t_{1}$ is the time instant when the output trajectory (in norm) enters in the interval $\left[\Delta_{i\left(t_{1}\right)}, \chi_{\theta_{i\left(t_{1}\right)}}\left(\Delta_{i\left(t_{1}\right)+1}\right)\right)$ with $i\left(t_{1}\right) \in\left\{\theta_{i\left(t_{0}\right)}-1, \theta_{i\left(t_{0}\right)}+1\right\}$. Assume that $t_{1}<+\infty$ (if $t_{1}$ is infinite, then the system is clearly forward complete and moreover the bound (7) holds) then from forward completeness of $\dot{\boldsymbol{x}}=\boldsymbol{f}_{\theta_{r}}(\boldsymbol{x}, \mathbf{d})$ and considering $\mathbf{d} \in M_{\mathbb{R}^{m}}$ we obtain that there exist $X_{0} \in \mathbb{R}_{+}$and $D_{0} \in \mathbb{R}_{+}$such that the solutions of (4), (23a) are defined over $\left[t_{0}, t_{1}\right)$ and, moreover, $\|\boldsymbol{x}\|_{\left[t_{0}, t_{1}\right)} \leqslant X_{0},\|\mathbf{d}\|_{\left[t_{0}, t_{1}\right)} \leqslant D_{0}$. Hence

$$
F_{0}=\sup _{\boldsymbol{x} \in N,|\boldsymbol{x}| \leqslant X_{0},|\mathbf{d}| \leqslant D_{0}}\left|\mathbf{f}_{\theta_{i\left(t_{0}\right)}}(\boldsymbol{x}, \mathbf{d})\right|<\infty
$$

which implies that for $|\boldsymbol{y}(t)|$ generated by system $\theta_{i\left(t_{0}\right)}$ to reach adjoining intervals $\left\{\left[\Delta_{\theta_{i\left(t_{0}\right)}-1}, \Delta_{\theta_{i\left(t_{0}\right)}}\right)\right.$ and $\left[\Delta_{\theta_{i\left(t_{0}\right)}+1}, \Delta_{\left.\theta_{i\left(t_{0}\right)}+2\right)}\right\}$ it is necessary a time proportional to the maximum 'speed' $F_{0}$ and $t_{1}-t_{0}$ units of time. Moreover, since $F_{0}<\infty$ necessarily $t_{1}>t_{0}$ therefore, the solutions of system (4), (23a) are defined and are continuous over $\left[t_{0}, t_{1}\right]$. Reconsidering the initial time to be $t_{1}$ and (4), (23a) we obtain that the solutions of the latter are defined for all $t \in\left[t_{1}, t_{2}\right)$ and satisfy

$$
\left|\boldsymbol{y}\left(t, \boldsymbol{x}\left(t_{1}\right), t_{1}\right)\right| \leqslant \beta_{\theta_{i\left(t_{1}\right)}}\left(\left|\boldsymbol{y}\left(t_{1}\right)\right|, t-t_{1}\right)+\gamma\left(S\left[\mathbf{d}, t_{1}, t_{2}\right]\right)
$$

and there exists finite numbers $X_{1} \in \mathbb{R}_{+}, D_{1} \in \mathbb{R}_{+}$and $F_{1} \in \mathbb{R}_{+}$such that

$$
\|\boldsymbol{x}\|_{\left[t_{1}, t_{2}\right)} \leqslant X_{1}, \quad\|\mathbf{d}\|_{\left[t_{1}, t_{2}\right)} \leqslant D_{1}, \quad F_{1}=\sup _{\boldsymbol{x} \in \mathcal{N},|\boldsymbol{x}| \leqslant X_{1},|\mathbf{d}| \leqslant D_{1}}\left|\mathbf{f}_{\theta_{i\left(t_{1}\right)}}(\boldsymbol{x}, \mathbf{d})\right|
$$

hence $t_{2}>t_{1}$. Repeating these arguments for arbitrary $j>0$ it is possible to prove the existence of $X_{j} \in \mathbb{R}_{+}, D_{j} \in \mathbb{R}_{+}$and $F_{j} \in \mathbb{R}_{+}$with properties

$$
\|\boldsymbol{x}\|_{\left[t_{j}, t_{j+1}\right)} \leqslant X_{j}, \quad\|\mathbf{d}\|_{\left[t_{j}, t_{j+1}\right)} \leqslant D_{j}, \quad F_{j}=\sup _{\boldsymbol{x} \in \mathcal{N},|\boldsymbol{x}| \leqslant X_{j},|\mathbf{d}| \leqslant D_{j}}\left|\mathbf{f}_{i_{i\left(t_{j}\right)}}(\boldsymbol{x}, \mathbf{d})\right|,
$$

so $t_{j+1}>t_{j}$. This implies right continuity of the switching signal $i(t)$ and forward completeness of the system (4), (23a).

The remainder of the proof follows along similar lines as the proof of Theorem 1: the estimates (11) and (12) continue to hold verbatim and if at $t_{k}$ we have $\left|\boldsymbol{y}\left(t_{k}\right)\right| \in\left[\Delta_{M}, \Delta_{M+1}\right)$ then (13) holds true. In view of (23a) we have either $\left|\boldsymbol{y}\left(t_{k}\right)\right|=\left|\boldsymbol{y}\left(t_{0}\right)\right|$ and $k=0$ or $\left|\boldsymbol{y}\left(t_{k}\right)\right|=\Delta_{M}$ (this is because according to the definition of the hysteresis supervisor, there is no dwell time); the bound (24) follows. The proof of statement (ii) follows as in Theorem 1 by replacing $T_{\min }$ with 0 ; finally, the proof of (iii) follows the same arguments as Theorem 1.

\subsection{On performance: the choice of $T_{q}$}

As a particular case-study of performance improvement we consider the scenario in which one seeks to increase the convergence rate of the output to zero (measured qualitatively via the $\mathscr{K} \mathscr{L}$ estimate). The following propositions define particular choices for the dwell-time functions depending on the settling time of the output trajectory, i.e. the time necessary for $\boldsymbol{y}(t)$ to reach a pre-defined compact (a prescribed tolerance).

Corollary 1

Let all conditions of Theorem 1 be satisfied and let $T_{q}$ satisfy

$$
\begin{aligned}
\beta_{\theta_{0}}\left(\Delta_{1}, T_{0}(s)\right) & =0.5 \Delta_{1}, \quad s \in\left[\Delta_{0}, \Delta_{1}\right) \\
\beta_{\theta_{q}}\left(s, T_{q}(s)\right) & =\chi_{\theta_{q-1}}\left(\Delta_{q}\right), \quad s \in\left[\Delta_{q}, \Delta_{q+1}\right), \quad q \in\{1, \ldots, M\} .
\end{aligned}
$$


Then, the time $T_{0.5 \Delta_{1}}$ that is required for $|\boldsymbol{y}(t)|$ to converge to the interior of the set $\left\{|\boldsymbol{y}| \leqslant 0.5 \Delta_{1}\right\}$ for the case $\mathbf{d}(t) \equiv 0$ can be estimated as follows (from (5a) $i(0)=r$ ):

$$
T_{0.5 \Delta_{1}} \leqslant \sum_{k=0}^{r} T_{k}\left(\Delta_{k+1}\right)
$$

If $\beta_{\theta_{q}}\left(s, T_{q}(s)\right)+\gamma\left(D_{q}\right)=\chi_{\theta_{q-1}}\left(\Delta_{q}\right), 0<\gamma\left(D_{q}\right)<\chi_{\theta_{q-1}}\left(\Delta_{q}\right), s \in\left[\Delta_{q}, \Delta_{q+1}\right)$ for $q \in\{1, \ldots, M\}$ and $\beta_{\theta_{0}}\left(\Delta_{1}, T_{0}(s)\right)+\gamma\left(D_{0}\right)=0.5 \Delta_{1}, 0<\gamma\left(D_{0}\right)<0.5 \Delta_{1}, s \in\left[\Delta_{0}, \Delta_{1}\right)$, then estimate (27) holds under disturbances $\mathbf{d} \in M_{\mathbb{R}^{m}}$ such that

$$
|\boldsymbol{y}(t)| \in\left[\Delta_{q}, \Delta_{q+1}\right), \quad q \in\{0, \ldots, M\}, t \in\left[t_{1}^{q}, t_{2}^{q}\right) \Rightarrow S\left[\mathbf{d}, t_{1}^{q}, t_{2}^{q}\right] \leqslant D_{q} .
$$

The relation (26) defines $T_{q}(\cdot)$ implicitly. For instance, for $\beta_{\theta_{q}}:=k s e^{-r}$ we obtain $T_{q}(s):=\ln (k s)-$ $\ln \left(\chi_{\theta_{q-1}}\left(\Delta_{q}\right)\right)$ while for $\beta\left(s, T_{q}(s)\right) \propto 1 / T_{q}(s)$ we obtain $T_{q}(s) \propto s$. Estimate (27) provides upper estimation on finite time of practical stabilization of the system with respect to set where $|\mathbf{y}(t)| \leqslant 0.5 \Delta_{1}$.

\section{Corollary 2}

Let all conditions of Theorem 1 be satisfied and expressions (25), (26) be valid. Assume that, for all $q \in\{0, \ldots, M\}$ and $s \in\left[\Delta_{q}, \Delta_{q+1}\right)$, there exists $t^{\prime} \in\left[T_{q}(s), T_{q}(s)+T_{q-1}\left(\chi_{\theta_{q-1}}\left(\Delta_{q}\right)\right)\right)$ such that $\beta_{\theta_{q}}(s, t) \geqslant \beta_{\theta_{q-1}}\left(\chi_{\theta_{q-1}}\left(\Delta_{q}\right), t-T_{q}(s)\right)$ for all $t \in\left[t^{\prime}, T_{q}(s)+T_{q-1}\left\{\chi_{\theta_{q-1}}\left(\Delta_{q}\right)\right\}\right)$. Then, for the case $\mathbf{d}(t) \equiv 0$ the output of the system (4), (5a) has the shortest time of convergence to $T_{0.5 \Delta_{1}}$ compared with that of any other system $\theta_{q}$ from the family (1), with initial output values in $\left[\Delta_{q}, \Delta_{q+1}\right)$.

Corollary 2 establishes conditions under which the output of system (4), (5a) has shorter settling time than any other subsystem in (1) over the domain $\left[\Delta_{q}, \Delta_{q+1}\right)$. The overall performance, i.e. for all $t$ is sub-optimal in the sense that it depends on the way the partitions in Figure 1 and the functions $T_{q}$ are chosen. The purpose of the following example is twofold: first, to illustrate the utility of Corollary 2 to choose the partitions determined by $\left\{\Delta_{q}\right\}$ and second, to provide an insight of the difficulty to obtain absolute optimality in the choice of the partitions and associated dwell-time functions.

\section{Example 1}

Consider a switched system of the form (4), (5a) with $\mathscr{I}=\{0,1\}$ satisfying Assumption 1 with functions $\beta_{0}$ and $\beta_{1}$. The goal is to find a set of conditions that lead to an improved rate of convergence. We have $M=2$ and the partition in Assumption 2 is $\mathbb{R}_{+}=\left[0, \Delta_{1}\right) \cup\left[\Delta_{1},+\infty\right)$. An appropriate choice of the threshold $\Delta_{1}$ leads to performance improvement qualitatively measured by a faster convergence of the $\mathscr{K} \mathscr{L}$ estimates. Since we are looking for the switching rule that minimizes the time of convergence let us introduce the functions $\mathscr{T}_{k}: \mathbb{R}_{+}^{2} \rightarrow \mathbb{R}_{+}$such, that $\beta_{k}\left(s, \mathscr{T}_{k}(s, \Delta)\right)=\Delta, s \in \mathbb{R}_{+}, \Delta \in \mathbb{R}_{+}, k=0,1$ (it is assumed that $\mathscr{T}_{k}(s, \Delta)=0$ for $\left.\beta_{k}(s, 0) \leqslant \Delta\right)$, i.e. $\mathscr{T}_{k}$ is the time that takes for the estimate on the output trajectories of system $k$ to reach a given level $\Delta$ for initial conditions $s$. For the level of interest here, i.e. $\Delta=\Delta_{1}$ assume first that $\mathscr{T}_{1}\left(s, \chi_{0}\left(\Delta_{1}\right)\right) \leqslant \mathscr{T}_{0}\left(s, \chi_{0}\left(\Delta_{1}\right)\right)$ for $s \geqslant \Delta_{1}$; in such case, it is reasonable to 'turn on dynamic mode 1 as long as the output trajectory remains within the upper interval $\left[\Delta_{1},+\infty\right)$ hence, we set $\theta_{1}=1$ and the system dynamics is defined by $\dot{\boldsymbol{x}}=\boldsymbol{f}_{1}(\boldsymbol{x}, \mathbf{d})$ on that interval. If on the other hand $\mathscr{T}_{0}(s, 0.5 \mathrm{~s}) \leqslant \mathscr{T}_{1}(s, 0.5 \mathrm{~s})$ for $s<\Delta_{1}$ it results reasonable to set $\theta_{0}=0$ hence, the system's dynamics is given by $\dot{\boldsymbol{x}}=\boldsymbol{f}_{0}(\boldsymbol{x}, \mathbf{d})$ as long as $|\boldsymbol{y}(t)| \in\left[0, \Delta_{1}\right)$. After the conditions of Corollary 2:

$$
\forall s \geqslant \Delta_{1} \quad \exists t^{\prime} \geqslant \mathscr{T}_{1}\left(s, \chi_{0}\left(\Delta_{1}\right)\right): \quad \beta_{1}(s, t) \geqslant \beta_{0}\left(\chi_{0}\left(\Delta_{1}\right), t-\mathscr{T}_{1}\left(s, \chi_{0}\left(\Delta_{1}\right)\right) \quad \forall t \geqslant t^{\prime}\right.
$$

so the switching condition to change from mode $1: \dot{\boldsymbol{x}}=\mathbf{f}_{1}(\boldsymbol{x}, \mathbf{d})$ to mode $0: \dot{\boldsymbol{x}}=\boldsymbol{f}_{0}(\boldsymbol{x}, \mathrm{d})$, is that $|\boldsymbol{y}(t)| \in\left[0, \Delta_{1}\right)$. It is important to remark that these conditions are only sufficient and the constant $\Delta_{1}$ is in general not the threshold that defines the switching rule which leads to absolute optimal performance (fastest possible convergence). However, relative improvement is always achievable following Corollary 2 .

Furthermore, let us consider the following optimization problem for the case of two systems. Let $1 \gg \varepsilon>0$ be a given tolerance level with respect to which to measure convergence, i.e. let us 
assume that it is required to find the shortest time for the output trajectories to satisfy $\left|\boldsymbol{y}\left(t, \boldsymbol{x}_{0}, 0\right)\right| \leqslant \varepsilon$. Then,

$$
\Delta_{\varepsilon}(s)=\underset{\Delta \leqslant s}{\arg \min }\left\{T_{1}(s, \Delta)+T_{0}(\Delta, \varepsilon)\right\}
$$

provides the optimal switching threshold we are looking for. If the solution of (29) is constant, i.e. if $\Delta_{\varepsilon}(s)=\widetilde{\Delta}$, then we set $\Delta_{1}=\widetilde{\Delta}$; if $\Delta_{\varepsilon}(s)$ varies with the initial state value then the choice $T_{1}(s)=T_{1}\left(s, \Delta_{\varepsilon}(s)\right)$ ensures that the output trajectory has an optimal convergence to the level $\varepsilon$ for all output trajectories of the switched system (4), (5a).

It may be apparent from this discussion that the difficulty of the latter optimization problem increases geometrically with respect to the number of systems, $N$, and partitions, $M$. Yet, satisfactory results may be obtained following sensible considerations on a case-by-case basis as it is further illustrated through particular applications in [11]. See also Section 5.

The next proposition gives guidelines for the choice of dwell-time functions under the hysteresis supervisor.

\section{Corollary 3}

Let all conditions of Theorem 2 hold and let $T_{q}$, for $0 \leqslant q \leqslant M$, be defined by (25), (26). Then, the time $T_{0.5 \Delta_{1}}$ that is required for $|\boldsymbol{y}(t)|$ to converge to the set $\left\{|\boldsymbol{y}| \leqslant 0.5 \Delta_{1}\right\}$ for the case $\mathbf{d}(t) \equiv 0$, satisfies

$$
T_{0.5 \Delta_{1}} \leqslant T_{r}\left(\Delta_{r+1}\right)+T_{r+1}\left(\Delta_{r+1}\right)+\sum_{k=0}^{r} T_{k}\left(\Delta_{k+1}\right) .
$$

An advantage of the hysteresis supervisor (23a) over the dwell-time supervisor (5a) is that the system's output under the dwell-time supervisor, may reach any interval $\left[\chi_{\theta_{q}}\left(\Delta_{q+1}\right), \Delta_{q+1}\right)$, $q \in\{0, \ldots, M\}$ before a switch occurs. In contrast to this, in the case of the hysteresis supervisor (23a) only adjoint intervals are considered; this results in a simpler analysis of the system's behaviour. Additionally, the supervisor (23a) ensures that only the system $\theta_{q}$ may become active for output values in the interval $\left[\Delta_{q}, \Delta_{q+1}\right.$ ) while for the dwell-time supervisor (5a) it is possible that the interval is reached during the dwell-time period by any (sub)system in (1). The latter allows to relax Assumption 1 for the hysteresis supervisor, as is explained in Remark 4.

On the other hand, as it is established in Corollaries 1 and 2 a proper choice of output depending dwell-time functions $T_{q}(\cdot)$ can ensure that the system possesses additional stability properties. For instance, the upper estimate (27) given in Corollary 1 for supervisor (5a) is better than the estimate provided by the conditions of Corollary 3 for supervisor (23a)). Additionally, for appropriate (large) values of dwell-time functions $T_{q}$ the switched system may admit an SIIOS-like estimate, see [20]. Another shortage of supervisor (23a) with respect to (5a) is that in general, high switching rates may be obtained. Nonetheless, in contrast to other hysteresis-based supervisors from the literature such as in [17], the hysteresis and partition properties do not imply restrictions on dwell-time values.

\section{EXAMPLE: HYBRID SYNCHRONIZATION OF LORENZ OSCILLATORS}

Consider the problem of master-slave synchronization of two Lorenz chaotic systems

$$
\text { master: }\left\{\begin{array}{l}
\dot{x}_{1}=\sigma\left(y_{1}-x_{1}\right)+d_{1}(t), \\
\dot{y}_{1}=x_{1}\left(\rho-z_{1}\right)-y_{1}+d_{2}(t), \\
\dot{z}_{1}=x_{1} y_{1}-\beta z_{1}+d_{3}(t),
\end{array}\right.
$$




$$
\text { slave: }\left\{\begin{array}{l}
\dot{x}_{2}=\sigma\left(y_{2}-x_{2}\right) \\
\dot{y}_{2}=x_{2}\left(\rho-z_{2}\right)-y_{2}+u_{1} \\
\dot{z}_{2}=x_{2} y_{2}-\beta z_{2}+u_{2}
\end{array}\right.
$$

where $\left(x_{i}, y_{i}, z_{i}\right) \in R^{3}, i=1,2$ are states, assumed measurable; $\mathbf{u}=\left(u_{1} u_{2}\right)$ are control inputs; $\mathbf{d}=\left(\begin{array}{lll}d_{1} & d_{2} & d_{3}\end{array}\right)$ are bounded disturbances; the parameters $\sigma>0, \rho>0$ and $\beta>0$ are identical for both systems and assumed known and such that the systems have a chaotic behaviour.

Boundedness of the trajectories of system (30) can be proved by using the Lyapunov function

$$
V=0.5\left[\sigma^{-1} x_{1}^{2}+y_{1}^{2}+\left(z_{1}-\rho\right)^{2}\right]
$$

which satisfies

$$
\dot{V} \leqslant-0.25 x_{1}^{2}-0.25 y_{1}^{2}-0.25 \beta\left(z_{1}-\rho\right)^{2}+4 \sigma^{-2} d_{1}^{2}+4 d_{2}^{2}+4 \beta^{-1} d_{3}^{2}+0.5 \rho^{2} \beta .
$$

In the sequel, we assume that the disturbances $\mathbf{d}$ are such that the limit set of the system (30) has nonempty intersection with the strange attractor existent for $\mathbf{d}=0$ (considering above it is always true for sufficiently small amplitudes of the disturbances).

The synchronization problem consists in making the states trajectories of the slave system follow those of the master. Hence, it may be recasted as a tracking control problem. It is largely motivated by application in secured telecommunication in which scenario the master system generates a chaotic carrier signal and transmits the encoded information. The receiver (slave system) is meant to synchronize, i.e. to mimic the behaviour of the master in order to recover the valuable information out of the carrier.

We briefly illustrate how hybrid control may be used to achieve controlled synchronization while minimizing the input energy. To that end, define the synchronization errors as $e_{1}=x_{1}-x_{2}$, $e_{2}=y_{1}-y_{2}, e_{3}=z_{1}-z_{2}$. The synchronization problem reduces to stabilizing the following system to zero via hybrid control.

$$
\begin{aligned}
& \dot{e}_{1}=\sigma\left(e_{2}-e_{1}\right)+d_{1}(t), \\
& \dot{e}_{2}=e_{1} \rho-x_{1} z_{1}+x_{2} z_{2}-e_{2}+d_{2}(t)-u_{1}, \\
& \dot{e}_{3}=x_{1} y_{1}-x_{2} y_{2}-\beta e_{3}+d_{3}(t)-u_{2} .
\end{aligned}
$$

We design control inputs that ensure input-to-state stability for system (32); in this case, systems (30), (31) are SIIOS with respect to the synchronization error $\mathbf{e}=\left(e_{1}, e_{2}, e_{3}\right)^{\top}$ as output and the input $\mathbf{d}$. In particular, we use the hysteresis supervisor (23a).

To comply with Assumptions 1 we start by designing controllers that achieve the synchronization goal. A first controller is a (global) cancellation control law given by

$$
u_{1}=\left(\rho+\sigma-2 \sqrt{(1-\lambda) \sigma}-z_{2}\right) e_{1}, \quad u_{2}=y_{2} e_{1}, \quad 0<\lambda<0.5
$$

for which system (32) has Lyapunov function $V(\mathbf{e})=0.5 \mathbf{e}^{\mathrm{T}} \mathbf{e}$ and

$$
\dot{V} \leqslant-\lambda \sigma e_{1}^{2}-\left(\sqrt{(1-\lambda) \sigma} e_{1}-e_{2}\right)^{2}-\beta_{3} e_{3}^{2}+\mathbf{e}^{T} \mathbf{d} \leqslant-\delta V+2 \sigma^{-1} d_{1}^{2}+0.5 d_{2}^{2}+2 \beta^{-1} d_{3}^{2},
$$

$\delta=\min \{\lambda \sigma, \lambda /(3 \lambda+1), \beta\}$ then, $\beta_{1}(s, t)=s e^{-0.5 \delta t}$ and Assumption 1 is satisfied for $i=1$.

The second control is based on a local linearization hence, it is to be applied for small errors $|\mathbf{e}| \leqslant \varepsilon$ for a given $\varepsilon>0$ :

$$
u_{1}=\alpha e_{1}, \quad u_{2}=0, \quad \alpha>0 .
$$

With controller (34) the system (32) can be rewritten as follows:

$$
\dot{\mathbf{e}}=\mathbf{A e}+\mathbf{D}(t), \quad \mathbf{A}=\left[\begin{array}{ccc}
-\sigma & \sigma & 0 \\
\rho-\alpha & -1 & 0 \\
0 & 0 & -\beta
\end{array}\right], \quad \mathbf{D}(t)=\left[\begin{array}{c}
d_{1}(t) \\
d_{2}(t)-x_{1}(t) z_{1}(t)+x_{2}(t) z_{2}(t) \\
d_{3}(t)+x_{1}(t) y_{1}(t)-x_{2}(t) y_{2}(t)
\end{array}\right],
$$


where $\|\mathbf{D}\| \leqslant\|\mathbf{D}\|+4 \varepsilon^{2}$ and $\mathbf{D} \in \mathscr{L}_{\infty}^{3}$ is a 'new' bounded disturbance. The system is linear time invariant and the matrix $\mathbf{A}$ is Hurwitz for properly chosen $\alpha$. The system with control (34) satisfies Assumption 1 locally: on $\{|\mathbf{e}| \leqslant \varepsilon\}$. If $\varepsilon<1$ the system is input-to-state stable with input $\mathbf{d}$.

A third controller is considered which consists in applying ... no input, i.e.

$$
u_{1}=0, \quad u_{2}=0 .
$$

The motivation for this control law is that both, master and slave, systems have solutions converging to compact sets with nonempty intersection. Since one system has chaotic trajectories that is dense in its chaotic attractor, there exists a time instant such that the distance between trajectories will be less than the strange attractor diameter (that can be evaluated numerically for the given parameters).

The controlled system corresponds to the open-loop dynamics (32), i.e.

$$
\begin{aligned}
\dot{\mathbf{e}} & =\mathbf{A}^{\prime} \mathbf{e}+\mathbf{D}^{\prime}(t), \quad \mathbf{A}^{\prime}=\left[\begin{array}{ccc}
-\sigma & \sigma & 0 \\
0 & -1 & 0 \\
0 & 0 & -\beta
\end{array}\right], \\
\mathbf{D}^{\prime}(t) & =\left[\begin{array}{l}
d_{1}(t), \\
d_{2}(t)-x_{1}(t) z_{1}(t)+x_{2}(t) z_{2}(t)+\rho\left[x_{1}(t)-x_{2}(t)\right], \\
d_{3}(t)+x_{1}(t) y_{1}(t)-x_{2}(t) y_{2}(t),
\end{array}\right]
\end{aligned}
$$

where the disturbance $\mathbf{D}^{\prime}$ is bounded (indeed it is composed by solutions of the system (30) and the system (31) with zero input, as it has been shown above these solutions are bounded functions of time) and $\mathbf{A}^{\prime}$ is Hurwitz, hence, Assumption 1 holds globally.

The three closed-loop systems may be written in the compact form (4). Let $I=\{1,2,3\}$ and consider the partition of $\mathbb{R}_{+}$defined in Assumption 2 with $M=3$ and $\theta_{0}=3, \theta_{1}=2, \theta_{2}=1$ and $\theta_{3}=3$. The practical motivation for such a partition is the following: we wish to diminish the amplitude of control energy, therefore, permanent application of the cancellation controller (33) is not desirable. Instead, we would like to switch to the linear local control (34) for relatively median values of the output errors (hence $\Delta_{2}=\varepsilon$ ). When synchronization errors are considerably small we may afford to switch off the control action, i.e. 'control' (35) is active for output errors smaller than $\Delta_{1} \ll \varepsilon \leqslant 1$. On the other hand, in the case when differences in the state trajectories are 'large' the use of the cancellation control law (33) may lead to large overshoots in control effort which is obviously undesirable since, in particular, it may cause actuator saturation. Hence, in this situation we also use control (35) and let the state trajectories of the forced and unforced Lorenz models (30) and (31) converge to their common strange attractor without control effort. In view of the latter, $\Delta_{3}$ is chosen proportional to the diameter of a sphere strictly containing the strange attractor which may be computed numerically.** Since the hysteresis supervisor is used, the dwell-time function $T_{q}$ can be skipped hence, to comply with Assumption 2 the values of $\Delta_{q}$ have to be assigned in accordance with the functions $\beta_{i}$ only. That is done below for a fixed set of parameters of the Lorenz systems.

\section{Example 2}

Let us consider a numerical example. Let $\sigma=10, \beta=8 / 3$ and $\rho=28 ; \lambda=0.1$ and $\alpha=28$; then

$$
\beta_{1}(s, t)=1.1 s \mathrm{e}^{-0.038 t}, \quad \beta_{2}(s, t)=\beta_{3}(s, t)=4.7 s \mathrm{e}^{-0.5 t}
$$

\footnotetext{
** We are not aware of any work computing analytically the 'size' of the Lorenz attractor.
} 
IMPROVING PERFORMANCE VIA SUPERVISORY CONTROL

Table I. Values of performance functionals for the case without disturbances.

\begin{tabular}{lrrrrrrrr}
\hline & \multicolumn{2}{c}{ Value of $J_{e}$} & & \multicolumn{2}{c}{ Value of $J_{a}$} & & \multicolumn{2}{c}{ Value of $J_{u}$} \\
\cline { 2 - 3 } & i.c. (36) & i.c. (37) & & i.c. (36) & i.c. (37) & & i.c. (36) & i.c. (37) \\
\hline Control (33) & 0.019 & 1.800 & & 0 & 0 & & 5.009 & 649.191 \\
Control (34) & 0.034 & 2.578 & & 0 & 0 & & 5.684 & 346.089 \\
Control (35) & 541.324 & 365.279 & & 518.694 & 350.101 & & 0 & 0 \\
Supervisor & 0.034 & 141.329 & & 0.009 & 0.009 & & 5.436 & 3.548 \\
\hline
\end{tabular}

Table II. Values of performance functionals for the case with disturbances (38).

\begin{tabular}{lrrrrrrrr}
\hline & \multicolumn{2}{c}{ Value of $J_{e}$} & & \multicolumn{2}{c}{ Value of $J_{a}$} & & \multicolumn{2}{c}{ Value of $J_{u}$} \\
\cline { 2 - 3 } & i.c. (36) & i.c. (37) & & i.c. (36) & i.c. (37) & & i.c. (36) & i.c. (37) \\
\hline Control (33) & 0.570 & 2.526 & & 0.864 & 0.864 & & 71.065 & 675.778 \\
Control (34) & 0.597 & 5.181 & & 0.335 & 0.335 & & 50.030 & 602.633 \\
Control (35) & 256.362 & 428.067 & & 474.852 & 375.061 & & 0 & 0 \\
Supervisor & 0.485 & 34.445 & & 0.317 & 0.317 & & 45.355 & 39.365 \\
\hline
\end{tabular}

and according to Assumption $2 \Delta_{1}=0.1, \Delta_{2}=1$ and $\Delta_{3}=5$ is an admissible choice. For illustration, we have performed simulations applying the hysteresis supervisor and the controls (33)-(35) individually. For the sake of comparison we use the following performance functionals:

$$
J_{e}=T^{-1} \int_{0}^{T}|\mathbf{e}(t)|^{2} \mathrm{~d} t, \quad J_{a}=10 T^{-1} \int_{.9 T}^{T}|\mathbf{e}(t)|^{2} \mathrm{~d} t, \quad J_{u}=T^{-1} \int_{0}^{T}|\mathbf{u}(t)|^{2} \mathrm{~d} t,
$$

where $T>0$ defines the length of simulations' windows. The functional $J_{e}$ describes the overall quality of synchronization in terms of the integral square error (ISE), the functional $J_{a}$ gives the ISE for the last tenth part of the simulation window, the functional $J_{u}$ estimates the input control energy. We use two sets of initial conditions (everywhere initial time is zero):

$$
\begin{aligned}
& x_{1}(0)=0.1, \quad y_{1}(0)=z_{1}(0)=0 ; \quad y_{2}(0)=1, \quad x_{2}(0)=z_{2}(0)=-1 \\
& x_{1}(0)=0.1, \quad y_{1}(0)=z_{1}(0)=0 ; \quad y_{2}(0)=10, \quad x_{2}(0)=z_{2}(0)=-10
\end{aligned}
$$

which correspond to 'small' and 'large' initial deviations of the systems (30) and (31), and disturbances

$$
d_{1}(t)=5 \sin (0.5 t), \quad d_{2}(t)=-5 \cos (0.1 t), \quad d_{3}(t)=2.5 \sin (t) .
$$

The simulation results are presented in Table I for the case without disturbances and in Table II for the disturbances (38) with $T=30 \mathrm{sec}$. Clearly, supervisory control ensures the best asymptotic performance while minimizing (relatively to the other two) control energy. While the overall transient is worse for large initial conditions (due to the application of control (35) which by itself does not solve the problem of synchronization) the 'asymptotic quality of synchronization expressed by the index $J_{a}$ is comparable to those provided by controls (33), (34) but with much smaller control effort, again, due to the use of controller (35).

For completeness, some plots are shown in Figures 2 and 3. The former presents the simulation results for the case with disturbances given by (38) and initial conditions given by (37) 
(a)
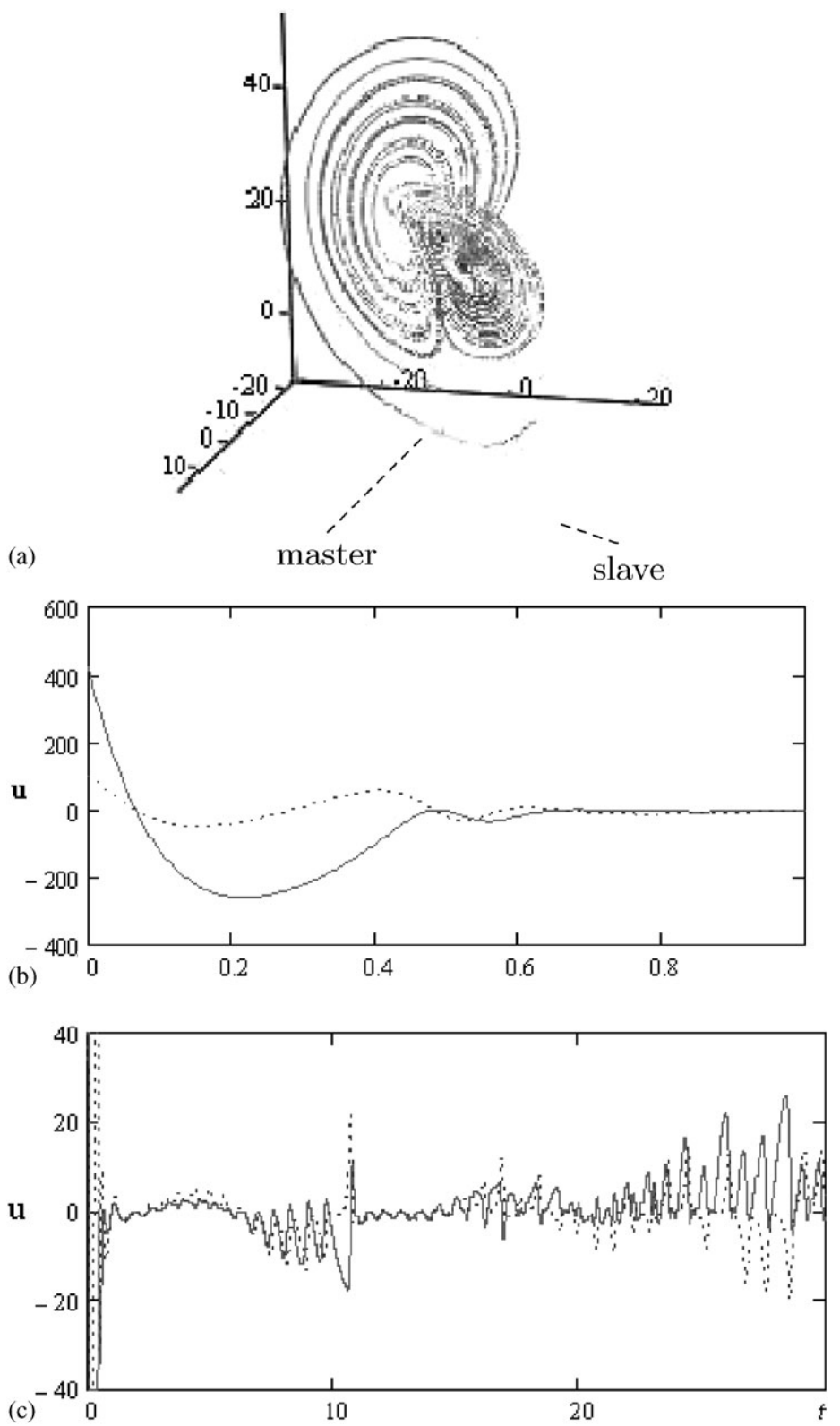

Figure 2. Lorenz systems under cancellation control: (a) Phase portraits; (b) control inputs (zoom of first sec); and (c) control inputs (zoom).

under control (33). Figure 3 depicts simulation results corresponding to supervisory control under the same conditions. More particularly, we show in Figures 2(a) and 3(a) the phase portraits of the master and slave systems under control (33) and hysteresis-based supervisory control, respectively. In Figure 2(b) and (c) we show the control efforts on different scales under control law (33); these may be compared with the supervisory control input depicted in Figure 3(b). As it is appreciated from the plots, the application of no control, i.e. (35) for the large initial errors results in a serious decreasing in the applied control energy $J_{u}$ and maximal amplitude of $|u(t)|$. The latter has the cost of 'high' peaks appreciated in the steady-state errors-see Figure 3 (b); yet the overall steady-state behaviour is comparable to that under controller (33) - see Figure 2(c). 
(a)
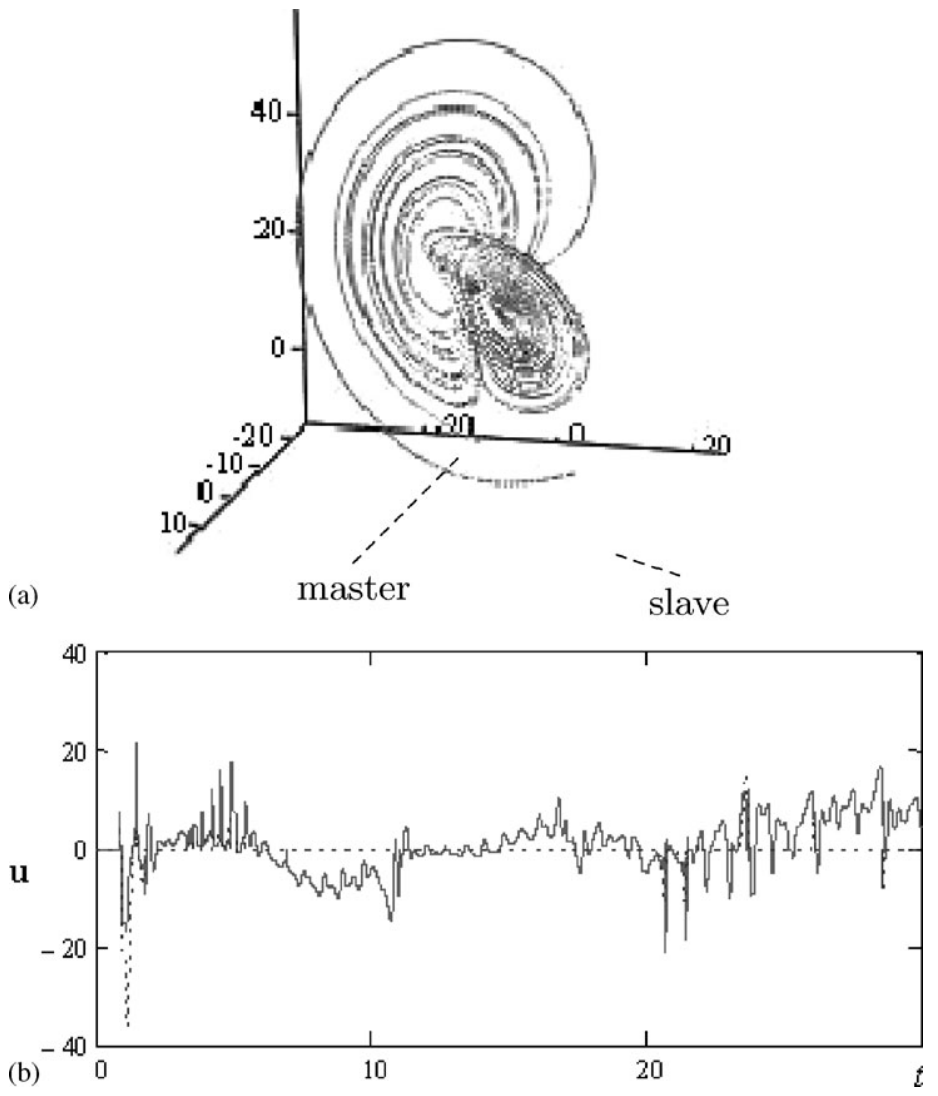

Figure 3. Lorenz systems under supervisory control: (a) Phase portraits and (b) control inputs.

\section{CONCLUSION}

We have addressed the problem of establishing input to output stabilization for a family of (switching) nonlinear systems with the aim of improving overall performance. Two approaches, on dwell-time and hysteresis supervisors, are proposed and discussed.

\section{ACKNOWLEDGEMENTS}

This work is supported by CNRS, EGIDE.

\section{REFERENCES}

1. Morse AS. Supervisory control of families of linear set-point controllers Part I: exact matching. IEEE Transactions on Automatic Control 1996; 41:1431-1996.

2. Liberzon D. Switching in systems and control. Systems and Control: Foundations and Applications. Birkhäuser: Boston, MA, U.S.A., 2003. ISBN: 0-8176-4297-8.

3. Goebel R, Sanfelice R, Teel A. Hybrid dynamical systems. IEEE Control Systems Magazine 2009; 29(2):28-93.

4. Hespanha J. Stabilization through hybrid control. In Encyclopaedia of Life Support Systems (EOLSS). Perspectives and Overview of Life Support Systems and Sustainable Development, Unbehauen H (ed.), Control Systems, Robotics, and Automation. EOLSS Publishers Ltd.: London, 2004.

5. Vu L, Chatterjee D, Liberzon D. Iss of switched systems and applications to switching adaptive control. Proceedings of the 44th IEEE Conference on Decision and Control, Seville, Spain, 2005; 120-125.

6. Xie W, Wen C, Li Z. Input-to-state stabilization of switched nonlinear systems. IEEE Transactions on Automatic Control 2001; 46:1111-1116.

7. Prieur C. Uniting local and global controllers with robustness to vanishing noise. Mathematical Control Signals Systems 2001; 14:172-2001. 
8. Pavlov A, van de Wouw N, Nijemeijer H. Output regulation of uncertain nonlinear systems. Systems and Control: Foundations and Applications (SC). Birkhäuser: Boston, MA, U.S.A., 2005.

9. Sontag ED, Wang Y. Notions of input-to-output stability. Systems and Control Letters 1999; 38:248-1999.

10. Hespanha JP, Santesso P, Stewart G. Optimal controller initialization between stabilizing controllers. Proceedings of the 46th IEEE Conference on Decision and Control, New Orleans, LA, U.S.A., 2007; 5634-5639.

11. Efimov D, Panteley E, Loria A. Robust output stabilization: improving performance via supervisory control. 2009. Available at: http://arxiv.org/abs/0906.0437v1.

12. Zheng Q, Wu F. Improving nonlinear control performance with a hybrid control strategy. Proceedings of the 46th IEEE Conference on Decision and Control, New Orleans, LA, U.S.A., 2007; 3208-3213.

13. Hespanha J, Morse A. Stability of switched systems with average dwell-time. Proceedings of the 38th IEEE Conference on Decision and Control, Phoenix, AZ, 1992; 655-2660.

14. Angeli D, Sontag ED. Forward completeness, unboundedness observability, and their Lyapunov characterizations. Systems and Control Letters 1999; 38:217-1999.

15. Sontag ED, Wang Y. Lyapunov characterizations of input-to-output stability. SIAM Journal on Control and Optimization 2001; 39:249-2001.

16. Morse AS. Control using logic-based switching. In Trends in Control, Isidory A (ed.). Springer: Berlin, 1995; 69-113.

17. Persis C, Santis R, Morse AS. Switched nonlinear systems with state-dependent dwell-time switching logic. Systems and Control Letters 2003; 50:302-2003.

18. Efimov DV. Uniting global and local controllers under acting disturbances. Automatica 2006; 42(6):549-554.

19. Prieur C, Astolfi A. Robust stabilization of chained systems via hybrid control. IEEE Transactions on Automatic Control 2003; 48(10):1768-1772.

20. Efimov D, Panteley E, Loria A. On input-to-output stability of switched nonlinear systems. Proceedings of the 17th IFAC World Congress, Seoul, South Korea, 2008; 3647-3642. 